\title{
New faunistic records and remarks on Dermestidae (Coleoptera). Part 21
}

\section{Новые фаунистические находки и замечания о жкуках-кожкеедах (Coleoptera, Dermestidae). Часть 21}

\author{
J. Háva*, A. Herrmann** \\ И. Гава*, А. Германн**
}

\begin{abstract}
* Forestry and Game Management Research Institute, Strnady 136, Praha 5 - Zbraslav CZ-156 00 Czech Republic. E-mail: jh.dermestidae@volny.cz.

* Научно-исследовательский институт охотничьего хозяйства, Стрнады 136, Praha 5 - 36 раслав CZ-156 00 Чешская Республика.

** Bremervörder Strasse 123, Stade D-21682 Germany. E-mail: herrmann@coleopterologie.de.

** Бременфёрдер штрассе 123, Штаде D-21682 ФРГ.
\end{abstract}

Key words: taxonomy, faunistics, new records, Coleoptera, Dermestidae.

Ключевые слова: таксономия, фаунистика, новые находки, Coleoptera, Dermestidae.

Abstract. 17 species of dermestid beetles are newly recorded from different localities of the world: Anthrenus (Anthrenodes) maculifer Reitter, 1881, Philippines: Palawan; A. (Anthrenus) kenyaensis Háva, 2004, Zimbabwe; Attagenus apicebrunneus Kalík, 1955, South Africa; A. fasciatus (Thunberg, 1795), Mali, Myanmar, Panama; A. posticalis Fairmaire, 1879, Jordan; Cryptorhopalum orbiculosum Reitter, 1881, Bolivia; C. pilosum Kirsch, 1870, Peru; Dermestes (Dermestes) ater DeGeer, 1774, Djibouti, Malaysia: Sabah; D. (Dermestinus) frischii Kugelann, 1792, Djibouti; D. (Dermestinus) gyl-lenhalii gyllenhalii Laporte de Castelnau, 1840, Bosnia-Herzegowina; D. (Dermestinus) maculatus DeGeer, 1774, Djibouti; D. (Dermestinus) undulatus Brahm, 1790, Portugal: Madeira; D. (Mon-tandonia) olivieri Lepesme 1939, Slovenia; Katkaenus spectaculus Háva, 2006, Laos; Orphinus (Orphinus) fulvipes (Guérin-Méneville, 1838), Guinea Equatorial, Panama; Paranovelsis siteki Háva, 2020, Croatia; Reesa vespulae (Milliron, 1939), Portugal: Madeira.

Резюме. Приводятся новые находки Anthrenus (Anthrenodes) maculifer Reitter, 1881 для Филиппин (Палаван); A. (Anthrenus) kenyaensis Háva, 2004 для Зимбабве; Attagenus apicebrunneus Kalík, 1955 для ЮАР; A. fasciatus (Thunberg, 1795) для Мали, Мьянмы и Панамы; A. posticalis Fairmaire, 1879 для Иордании; Cryptorhopalum orbiculosum Reitter, 1881 для Боливии; C. pilosum Kirsch, 1870 для Перу; Dermestes (Dermestes) ater DeGeer, 1774 для Джибути и Малайзии $(\mathrm{Ca}-$ бах); D. (Dermestinus) frischii Kugelann, 1792 для Джибути; D. (Dermestinus) gyllenhalii gyllenhalii Laporte de Castelnau, 1840 для Боснии и Герцоговины; D. (Dermestinus) maculatus DeGeer, 1774 для Джибути; D. (Dermestinus) undulatus Brahm, 1790 для Португалии (Мадейра); D. (Montandonia) olivieri Lepesme, 1939 для Словении; Katkaenus spectaculus Háva, 2006 для Лаоса; Orphinus (Orphinus) fulvipes (Guérin-Méneville, 1838 д для Экваториальной Гвинеи и Панамы; Paranovelsis siteki Háva, 2020 для Хорватии; Reesa vespulae (Milliron, 1939) для Португалии (Мадейра).

\section{Introduction}

The knowledge about the distribution of many species within the family Dermestidae is still incomplete. There are a lot of gaps in our understanding of the occurrence of many species. Thus, each paper, which supplements our knowledge in this field, is valuable. In the present paper, 17 species belonging to the family Dermestidae (Coleoptera) are recorded and 3 three species are discussed. The article is a continuation of a series of 20 previous articles [e. g. Háva, Herrmann 2016, 2018, 2019a, b, 2020].

\section{Material and methods}

Species are arranged in alphabetical order, the nomenclature and zoogeography follow the catalogue of Háva [2015, 2020a].

The following abbreviations refer to the collections, in which the examined materials are deposited: AHEC Andreas Herrmann, private collection, Stade, Germany; BMNH - British Museum Natural History, London, United Kingdom; FAPC - Antonio Miguel Franquinho Aguiar, private collection, Madeira, Spain; ICLAM Insect Collection Laboratório Agrícola da Madeira, Madeira, Spain; ISNB - National Belges d'Insectes et d'Arachnides, Bussels, Belgium; JHAC — Jiří Háva, Private Entomological Laboratory \& Collection, Únětice u Prahy, Prague-West, Czech Republic; JLPC — Josiane Lips, private collection, Lyon, France; JMLC Jean-Michel Lemaire, private collection, Alpes-Maritimes, France; MIUP - Universidad de Panamá, Museo de Invertebrados «G.B. Fairchild», Panamá, Panama; MZUF - Museo Zoologico «La Specola», Firenze, Italy; TMSA - Ditsong National [Transvaal Museum] Museum of Natural History, Pretoria, South Africa; ZMUB - Humbolt-Universität [Zoologisches Museum], Museum für Naturkunde, Berlin, Germany; WWPC - Wolfgang Waitzbauer, private collection, Drosendorf, Austria. 


\section{Undescribed taxa}

In the Museum für Naturkunde, Berlin, Germany (ZMUB) the authors determined three unpublished specimens:

Anthrenus (Nathrenus) biskrensis Reitter, 1887 Figs 1-2.

= Anthrenus repandus Erichson

Material. Italy, «Hist.-Coll (Coleoptera), Nr. 9298, Atbrenus repandus Erichs., Sicil., Zell. Zool. Mus. Berlin/ 9298 / repandus Er, Sicil. Zell.» - 1 spec., (ZMUB).

Remarks. The specimen from Sicilia labelled as Anthrenus repandus Erichson is undescribed. According to the characters it belongs to Anthrenus (Nathrenus) biskrensis Reitter, 1887.

\section{Orphinus (Orphinus) terminalis \\ (Sharp in Blackburn et Sharp, 1885) Figs 3-4.}

= Trogoderma vagans Fauvel.

Material. New Caledonic, «N. Caled / Trogoderma vagans Fauv. N. Caledon. / Trogoderma vagans Fvl.» «Coll L.W. Schaufuss» - 1 spec., (ZMUB).

Remarks. The specimen from New Caledonia labelled as Trogoderma vagans Fauvel is undescribed and not mentioned in Fauvel [2003]. According to the characters it belongs to Orphinus (Orphinus) terminalis (Sharp in Blackburn et Sharp, 1885).

Distribution. Species known from Hawaiian Is., Philippines, Cook Is., Gilbert Is., South Mariana Is., Marshall Is., Samoa Is., Upolu Is. [Háva, 2015], new for New Caledonia.

\section{Orphinus sp.}

Figs 5-6.

Material. New Caledonic, «N. Caled. / Trogoderma migrator Fauv. N. Caledon. / Attagenus ?, N. Caledon. / Trogoderma migrator Fvl.» «Coll - L.W. Schaufuss» - 1 spec., (ZMUB).

Remarks. The specimen from New Caledonia labelled as Trogoderma migrator Fauvel is undescribed and not mentioned in Fauvel [1903]. According to the torso (elytrae and abdomen) the specimen probably belongs to the genus Orphinus Motschulsky.

\section{Faunistics}

\section{Dermestinae}

Dermestini

Dermestes (Dermestes) ater DeGeer, 1774

Material. Malaysia, N. Borneo, Sabah, (nr. Kpg. Bunsit), 1.05.1984, Masaaki Nishikawa leg., 1 female, A. Herrmann det. (AHEC); Djibouti, about $5 \mathrm{~km} \mathrm{SW}$ Obock, surr. Campement Oubouki, 24-27.1.2016, P. Agnelli, A. Nistri, A. Ugolini leg. (nMagazz.3076) - 1 spec., J. Háva det., (MZUF).

Distribution. Cosmopolitan species, first concrete record from Malaysia: Sabah and Djibouti.

\section{Dermestes (Dermestinus) frischii Kugelann, 1792}

Material. Djibouti, a W of Obock, $\left(11^{\circ} 57^{\prime} 39^{\prime \prime} \mathrm{N}\right.$, 4314'52" E), 21.2.2013, P. Agnelli, A. Nistri \& A. Ugolini leg., Mag. N. 2803 / Diibouti, nella testa in decomposizione di Isnemia albicauda Cuvier, 1829 (Herpestidae, Carnivora) 3 spec., J. Háva det., (2 MZUF, 1 JHAC).

Distribution. Cosmopolitan species, first concrete record from Djibouti.

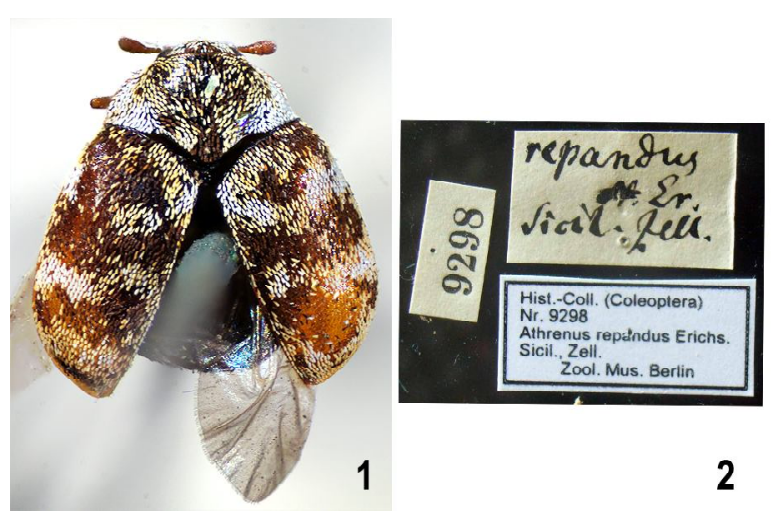

Figs 1-2. Anthrenus (Natbrenus) biskrensis (= Anthrenus repandus): 1 - habitus, dorsal aspect; 2 - labells.

Pис. 1-2. Antbrenus (Nathrenus) biskrensis (= Anthrenus repandus): 1 - внешний виА, сверху; 2 - этикетки.
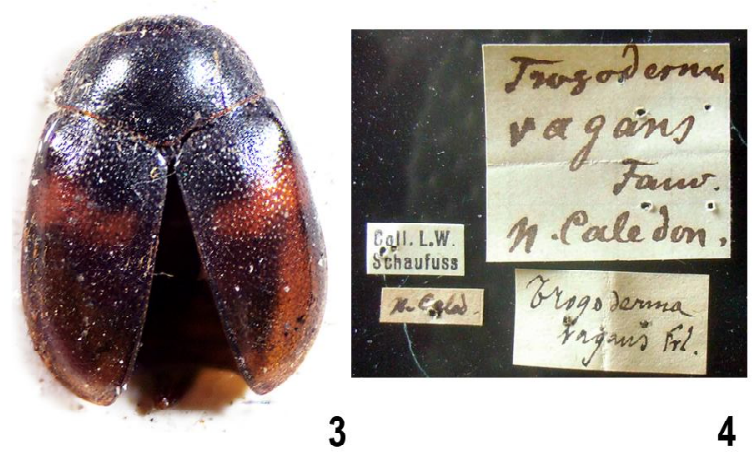

Figs 3-4. Orphinus (Orphinus) terminalis (Sharp in Blackburn et Sharp, 1885) (= Trogoderma vagans Fauvel): 3 habitus, dorsal aspect; 4 - labells.

Рис. 3-4. Orphinus (Orphinus) terminalis (Sharp in Blackburn et Sharp, 1885) (= Trogoderma vagans Fauvel): 3 внешний виА, сверху; 4 - этикетки.
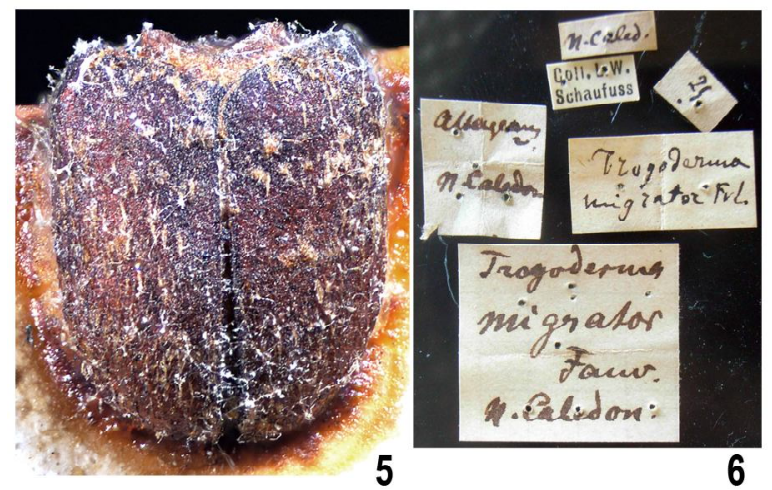

Figs 5-6. Orpbinus sp. (= Trogoderma migrator Fauvel): 5 - habitus, dorsal aspect; 6 - labells.

Рис. 5-6. Orphinus sp. (= Trogoderma migrator Fauvel): 5 - внешний виА, сверху; 6 - этикетки.

Dermestes (Dermestinnus) gyllenhalii gyllenhalii Laporte de Castelnau, 1840

Material. Bosnien-Herzegowina, Mostar/Neretva-Ufer, 18.08.1977, G. A. Lohse leg. - 19, A. Herrmann det., (AHEC). 
Distribution. Species known from Europe, Caucasus, Iran, Kazakhstan, Russia, first concrete record from BosnaHerzegovina.

Dermestes (Dermestinus) maculatus DeGeer, 1774

Material. Djibouti, Tadjoura Reg., Bankouwale (11\%49' N, 42 40' E), 28.II-1.III.2013, leg. Agnelli, Nistri, Ugolini Museo Zoologico «La Specola» Num. Mag. 2951 - 1 spec., J. Háva det., (MZUF).

Distribution. Cosmopolitan species, first concrete record from Djibouti.

\section{Dermestes (Dermestinus) undulatus Brahm, 1790}

Material. Portugal, ICLAM05773: multi-funnel pheromone trap on Pinus pinaster, Boca da Corrida, Cвmara de Lobos, Madeira Island, 32.7015706, -16.9920528, 1000 m, 16.07.2015, A. Aguiar leg. - 1 spec., (ICLAM); ICLAM05862: multi-funnel pheromone trap on Pinus pinaster, Sucata, Santana, Madeira Island, 32.7896892, -16.8897118, 682 m, 27.08.2015, A. Aguiar leg. - 1우, (ICLAM); cFA1763: multi-funnel pheromone trap on Pinus pinaster, Loural, Sro Vicente, Madeira Island, 32.7680614, -17.0342871, 534 m, 2.09.2015, A. Aguiar leg. $10^{7}$, (FAPC).

Distribution. Holarctic species, new to Portugal: Madeira Island.

Dermestes (Montandonia) olivieri Lepesme, 1939

Material. Slowenien, Rovinj/Istrien, IX.1967, Just leg. 1 spec., A. Herrmann det., (AHEC).
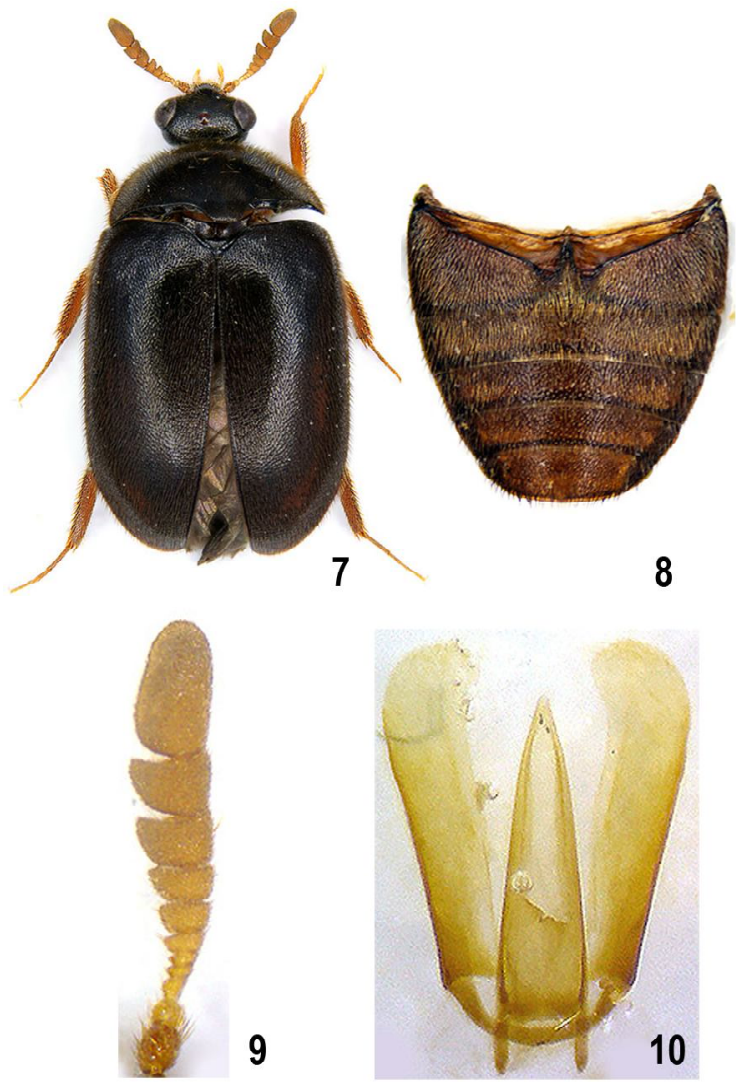

Figs 7-10. Katkaenus spectaculus Háva, 2006 (Laos): 9 habitus, dorsal aspect; 10 - antenna of male; 11 - abdomen; 12 - male genitalia.

Pис. 7-10. Katkaenus spectaculus Háva, 2006 (Laos): 9 внешний вид; 10 - усик, самещ; 11 - брюшко; 12 гениталии, самец.
Distribution. Species known from C, S Europe, Armenia, Georgia, Cyprus, Ukraine, Turkey, Tunisia, Iran, Lebanon, Russia, Syria and Turkmenistan, first concrete record from Slovenia.

\section{Attageninae}

Attagenus apicebrunneus Kalík, 1955

Material. South Africa, «S.Afr., Cape Prov., Vanzyisrus $167 \mathrm{~km} \mathrm{W,} \mathrm{26.3.1990} \mathrm{/} \mathrm{on} \mathrm{flowers,} \mathrm{leg.} \mathrm{J.} \mathrm{Klimaszewski»} \mathrm{-} 7$ spec., J. Háva, A. Herrmann det., (5 TMSA, 2 AHEC).

Distribution. Species known from Kenya; Malawi; Tanzania; Zambia; Zimbabwe, new for South Africa.

Remarks. The specimens were already recorded errously as Attagenus donckieri Pic, 1916 [Háva, Herrmann 2018], but now it has turned out that the record based on a wrong identification. The concerning specimens actually belong to Attagenus apicebrunneus Kalík, 1955.

\section{Attagenus fasciatus (Thunberg, 1795)}

Material. Myanmar (Burma), Bagan, Irrawaddy - Ufer, 7.02.1998, Ernst Heiss leg., 1 spec., A. Herrmann det., (AHEC); República de Panamá, Provincia Panamá, San Miguelito, Villa Guadalupe, 25.enero 1980, leg. E. Polo - 1 spec., J.M. Kingsolver det., (MIUP); Mali, Bamako Soudan, 10.05.1946, ex coll. Bettinger - 12 spec., A. Herrmann det., (10 ISNB, 2 JHAC).

Distribution. Cosmopolitan species, first concrete records from Mali, Myanmar and Panama.

\section{Attagenus posticalis Fairmaire, 1879}

Material. Jordania, Wadi Rum Sand desert, 29 $34^{\prime}$ N, $35^{\circ} 26^{\prime}$ E, I-2014, leg. Waitzbauer - 107, 20ㅇ, A. Herrmann det., (2 AHEC, 1 WWPC).

Distribution. Species known from Spain, Algeria, Egypt, Eritrea, Ethiopia, Mauretania, Morocco, Niger, Senegal, Sudan, Tunisia, Israel, Kuwait, Oman, Qatar, Saudi Arabia, Syria, United Arab Emirates and Yemen, new to Jordan.

\section{Katkaenus spectaculus Háva, 2006}

Figs $7-10$.

Material. Laos, Xe Bang Fai/Khammouan, Feb. 1919, leg. Terry Bolger - 90 $0^{7}$, J. Háva det., (3 AHEC, 1 JHAC, 4 JMLC, 1 JLPC).

Distribution. Species known from India: Tamil Nadu, new to Laos.

\section{Paranovelsis siteki Háva, 2020}

Material. Croatia, «Požega [Croatia, 4520' N 1741' E, $145 \mathrm{~km} \mathrm{SW}$ of Zagreb], 20.5.1914» - 10", J. Háva det., coll. A. Olexa., (JHAC).

Distribution. Species described from Greece (Háva 2020b), new to Croatia.

\section{Megatominae Anthrenini}

Anthrenus (Anthrenodes) maculifer Reitter, 1881

Material. Philippines, Palawan, San Vicente, Oct. 2019, local collector -2 spec., J. Háva det., (JHAC).

Distribution. Species known from China, India, Indonesia, Japan, Laos, Malaysia, Myanmar, Nepal, Taiwan, Thailand and Vietnam, new to Philippines: Palawan.

Anthrenus (Anthrenus) kenyaensis Háva, 2004

Material. Zimbabwe, Salisbury, Mashonaland, Marshall, 1915.334 - 1 spec., J. Háva det., (BMNH).

Distribution. Species known from Kenya, South Africa: Transvaal, Tanzania, new to Zimbabwe. 


\section{Megatomini}

Cryptorhopalum orbiculosum Reitter, 1881

Material. Bolivia, Buena Vista/Santa Cruz/Ichilo, 10.2.2000, W. B. Warner leg. - 1 spec., A. Herrmann, J. Háva det., (AHEC).

Distribution. Species known from Antilles Isl.: St. Lucia, Brazil, Colombia, French Guiana, Panama, Peru, Tobago and Venezuela, new to Bolivia.

\section{Cryptorhopalum pilosum Kirsch, 1870}

Material. Peru, Dep. Cuzco, $15 \mathrm{~km} \mathrm{~S}$ Quillabamba, 2 $\mathrm{km}$ W Chaullay, 3.06.2007, Robert Constantin leg. - 10 A. Hermann det., (AHEC); Peru, reg. Huánuco, Tingo Maria $40 \mathrm{~km} \mathrm{NE} \mathrm{Abra} \mathrm{Divisoria,} \mathrm{Rio} \mathrm{Azul,} \mathrm{11.04.2013,} \mathrm{Robert}$ Constantin leg. - 2 spec., A. Herrmann det., (AHEC).

Distribution. Species known from Colombia, Costa Rica, Nicaragua, Panama and Trinidad, new to Peru.

Orphinus (Orphinus) fulvipes (Guérin-Méneville, 1838)

Material. Guinea Espanol, Evinayong, Dr. L. Báguena - 20 spec., A. Herrmann det., (AHEC); Fernando Poo, Sta. Isabel, VII.1919 Escalera - 1 spec., A. Herrmann det., (AHEC); República de Panamá, Provincia Panamá, Orilla Lago Alajuela, 27.Oct.1978, leg. A. Arauz - 1 spec., R. Cambra det. (MIUP); República de Panamá, Las Cumbres, 6. Feb.-5. Mar. 1985, leg. H. Wolda (light trap) - 1 spec, J.M. Kingsolver det., (MIUP).

Distribution. Cosmopolitan species, new to Guinea Equatorial and Panama.

\section{Reesa vespulae (Milliron, 1939)}

Material. Portugal, ICLAM03815: collected inside lab building, Ribeirinha, Camacha, Madeira Island, 32.6728371, -16.8473992, 649 m, 28.06.2011, A. Aguiar leg., 1 spec., A. Herrmann revid., (ICLAM).

Distribution. Species known from Europe, north Africa, Canada, U.S.A., Mexico, Argentina, Chile, Afghanistan, Chi- na, Japan, Korea, Russia, Australia and New Zealand, new to Portugal: Madeira Island.

\section{Acknowledgements}

We are indebted to all colleagues and collectors for the chance to study their material. The paper was supported by the Ministry of Agriculture of the Czech Republic, institutional support MZE-RO0118.

\section{References}

Fauvel A. 1903. Faune analytique des Coléoptéres de la Nouvelle Calédonie // Revue Entomologique. Vol.22. P.203-379.

Háva J. 2015. World Catalogue of Insects. Volume 13. Dermestidae (Coleoptera). Leiden/Boston: Brill, xxvi +419 p.

Háva J. 2020a. Dermestidae World (Coleoptera). World Wide Web electronic publication (open in 2004): http:// www.dermestidae.wz.cz (version 2018, update January 2020)

Háva J. 2020b. A new species of the genus Paranovelsis Casey, 1900 from Greece (Coleoptera: Dermestidae: Attageninae) // Studies and Reports, Taxonomical Series. Vol.16. No.1. P.79-83.

Háva J., Herrmann A. 2016. New faunistic records and remarks on Dermestidae (Coleoptera) - Part $16 / /$ Folia Heyrovskyana. Ser.A Vol.25. No.2. P.4-14.

Háva J., Herrmann A. 2018. New faunistic records and remarks on Dermestidae (Coleoptera). Part 17 // Folia Heyrovskyana. Ser.A. Vol.26. No.1. P.13-18.

Háva J., Herrmann A. 2019a. New faunistic records and remarks on Dermestidae (Coleoptera) - Part $18 / /$ Studies and Reports, Taxonomical Series. Vol.15. No.1. P.41-53.

Háva J., Herrmann A. 2019b. New faunistic records and remarks on Dermestidae (Coleoptera). Part 19 // Natura Somogyiensis. Vol.33. P.27-36.

Háva J., Herrmann A. 2020. New faunistic records and remarks on Dermestidae (Coleoptera). Part 20 // Natura Somogyiensis. Vol.34. P.9-20. 